\title{
How "volatile" is the protection provided by inhalational anesthetics?
}

Beck-Schimmer, Beatrice ; Shafer, Steven L

DOI: https://doi.org/10.1097/ALN.0000000000001121

Posted at the Zurich Open Repository and Archive, University of Zurich ZORA URL: https://doi.org/10.5167/uzh-127097

Journal Article

Published Version

Originally published at:

Beck-Schimmer, Beatrice; Shafer, Steven L (2016). How "volatile" is the protection provided by inhalational anesthetics? Anesthesiology, 124(6):1213-1214.

DOI: https://doi.org/10.1097/ALN.0000000000001121 


\title{
How "Volatile" Is the Protection Provided by Inhalational Anesthetics?
}

\author{
Beatrice Beck-Schimmer, M.D., Steven L. Shafer, M.D.
}

CME

This article has been selected for the ANESTHESIOLOGY CME Program. Learning objectives and disclosure and ordering information can be found in the CME section at the front of this issue.

In $\mathrm{N}$ this issue of Anesthesiology, Uhlig et al. ${ }^{1}$ compare morbidity and mortality with volatile anesthetics versus total intravenous anesthesia in a comprehensive meta-analysis of 68 published randomized controlled trials (RCTs) that included 7104 patients. The authors found that outcomes in patients undergoing cardiac surgery were different from outcomes in other patient groups, and they stratified their results accordingly. The primary endpoint was mortality, defined as the longest mortality reported, in-hospital or 30-day mortality. The secondary endpoints were pulmonary and other complications. Volatile anesthetics were associated with reduced mortality and postoperative complications in patients undergoing cardiac surgery when compared with intravenous anesthesia. However, the choice of anesthetic did not influence mortality or postoperative complications for other types of surgery.

We congratulate the authors for this fine analysis. They carefully researched three databases, complying meticulously with the Preferred Reporting Items for Systematic Reviews and Meta-Analyses guidelines. The topic addressed is scientifically interesting and clinically relevant. The results bring together a large number of trials addressing the question of organ protection. Given the comprehensiveness of this analysis, the high quality of the RCTs analyzed, and the meticulous statistical treatment of the results, perhaps Uhlman et al. have published the definitive analysis. However, the authors suggest that more research is needed.

For a proper interpretation of studies suggesting immunomodulation by volatile anesthetics improving clinical outcome, the following issues deserve careful evaluation:
1. Some studies have been performed in scenarios of a "hit," e.g., ischemia-reperfusion injury in transplantation or cardiac surgery with extracorporeal circulation, while in other studies, such events remain elusive. One has to acknowledge the fact that there is no protection from an injury, if there is no injury. Additionally, the degree of injury certainly matters, which could explain the findings of the current study. ${ }^{1}$

2. The mode of application of volatile anesthetics may play an important role. The authors did not differentiate between administering volatile anesthetics for the entire duration of surgery and a "pre-conditioning" application, where the anesthetic was applied right before the ischemic injury. Obviously, such an application is very clear during cardiac surgery, but much less so in other types of surgery that do not involve a specific ischemic challenge. Subtle details like these may well impact outcome.

3. The authors have reasonably grouped together all volatile anesthetics. While that makes sense, we do not know if isoflurane, sevoflurane, and desflurane are equally protective. Uhlig et al. ${ }^{1}$ point out that sevoflurane and desflurane seemed more protective than isoflurane in cardiac surgery patients.

4. We should bear in mind that most of the clinical studies have focus on biomarkers such as troponin, transaminases, creatinine, inflammatory mediators, or markers of cell death. The power analysis has been performed with regard to such surrogate markers, while the studies with clinically relevant outcomes are few and far between.

5. Additionally, we notice that even when relevant clinical outcomes are reported, they are frequently not presented

\footnotetext{
Image: J. P. Ratbmell.

Corresponding article on page 1230.
}

Accepted for publication March 9, 2016. From the Institute of Anesthesiology, University Hospital Zurich, Zurich, Switzerland (B.B.-S.); Department of Anesthesiology, University of Illinois Chicago, Chicago, Illinois (B.B.-S.); and Department of Anesthesiology, Perioperative and Pain Medicine, Standford University Medical Center, Stanford, California (S.L.S.). 
using a well-defined scoring system. The definition of "pulmonary infection" for example seems to vary from study to study and does not allow serving as an operation endpoint. 6. Since the RCTs in this study compared volatile anesthetics to propofol, we naturally attribute differences in outcomes to the anesthetics. However, the strength of the RCT design is that differences in outcome are causally linked to assignment to a treatment group. The RCT design does not tell us why one group did better than the other. The authors naturally attributed the better outcomes with inhaled anesthetics for cardiac surgery to the pharmacology of volatile anesthetics. Maybe, but that is a guess. The better outcomes might be caused by differences in physician experience with the assigned treatment, differences in anesthetic depth between the two approaches, differences in other drugs (e.g., how many patients get inhaled anesthetics and remifentanil?), effects of propofol on the bypass circuit, ${ }^{2}$ or other nonpharmacologic differences between the two groups. We know that in the published RCTs for cardiac surgery, assignment to volatile anesthesia gives better outcomes than assignment to propofol. We do not know why.

Several limitations are discussed by the authors. First, negative results are less likely to be published. This truism remains a major problem with clinical trials and refuses to go away. ${ }^{3}$ We cannot blame journals, readers, or authors for this. It is intrinsic to science that a small trial with a positive result is exciting and gets published, but an equally small trial with no result has no chance of publication. Consider the recent demonstration of gravity waves, based on four observations (two events at two observatories). ${ }^{4}$ This is likely one of the most important scientific discoveries in human history, and certainly within our lifetime. However, there is no chance that more than 1000 coauthors would have chosen to write a paper if there had been no observations for several months at the two observatories. We rightly ask authors, and journals, to be more receptive to negative trials. However, it is the nature of science that it takes only one observation to prove that something exists, but no amount of data can prove something does not exist.

The authors also point out that elements of anesthesia such as opioids, local anesthetics used for regional anesthesia, statins, etc., are protective as well and constitute powerful confounders. These are rarely reported in sufficient detail in RCTs for a meta-analysis to fully account for these confounding variables. For example, remifentanil infusions are commonly used with propofol, but will be only rarely used with an inhaled anesthetic. Perhaps the worse outcome in the cardiac surgery patients with propofol is because of the remifentanil. We are not seriously suggesting this, but simply using this to observe that confounding variables involving other drugs are likely not to be randomly divided between the two techniques.

Uhlig et al., an experienced group of researchers in the field of organ protection, summarize their conclusions with the adage "further studies are warranted." Is this really what we need? The two authors of this editorial have different opinions about this conclusion. One believes that it would be quite reasonable to use this meta-analysis as a basis to design a definitive clinical trial (B.B.S.). If we want to have evidence-based protocols for the patients entrusted to us for optimal care, we urgently need large RCTs focusing on well-defined clinical endpoints such as complications and mortality. Or, stated differently, we still have not reached evidence level I yet. ${ }^{5}$ The enthusiasm of the second author of the editorial, however, is tempered by many studies summarized in the systematic review that demonstrate little difference in outcome between propofol and volatile anesthesia (S.L.S.). Given the work that has been done, and the intrinsic limitations of clinical studies (e.g., item 6 above), the conclusion is slightly different: "are further studies warranted?"

\section{Competing Interests}

The authors are not supported by, nor maintain any financial interest in, any commercial activity that may be associated with the topic of this article.

\section{Correspondence}

Address correspondence to Dr. Beck-Schimmer: beatrice. beckschimmer@uzh.ch or beatrice.beck@usz.ch

\section{References}

1. Uhlig C, Bluth T, Schwarz K, Deckert S, Heinrich L, De Hert S, Landoni G, Neto A, Schultz M, Pelosi P, Schmitt J, Gama de Abreu M: Effects of voaltile anesthetics on mortality and postoperative pulmonary and other complications in patients undergoing surgery: A systematic review and meta-analysis. Anesthesiology 2016; 124:1230-45

2. Arya VK, Kumar A, Thingnam SK: Propofol infusion into the pump during cardiopulmonary bypass: Is it safe and effective? J Cardiothorac Vasc Anesth 2004; 18:122-3

3. Dwan K, Altman DG, Arnaiz JA, Bloom J, Chan AW, Cronin E, Decullier E, Easterbrook PJ, Von Elm E, Gamble C, Ghersi D, Ioannidis JP, Simes J, Williamson PR: Systematic review of the empirical evidence of study publication bias and outcome reporting bias. PLoS One 2008; 3:e3081

4. Abbott B: Observation of gravitational waves from a binary black hole merger. Phys Rev Lett 2016; 116:061102

5. Sackett DL: Rules of evidence and clinical recommendations on the use of antithrombotic agents. Chest 1989; 95:2S-4S 